\title{
Un modelo simple para describir la dinámica de la biomasa del camarón nailon Heterocarpus reedi en Coquimbo, Chile
}

\author{
Eduardo P. Pérez ${ }^{1,2}$ \\ ${ }^{1}$ Departamento de Biología Marina, Facultad de Ciencias del Mar \\ Universidad Católica del Norte, Casilla 117, Coquimbo, Chile \\ ${ }^{2}$ Centro de Estudios Avanzados en Zonas Áridas, CEAZA, Coquimbo
}

\begin{abstract}
RESUMEN. Entre septiembre de 1997 y agosto de 2000 se realizó un seguimiento diario de la dinámica espacial y temporal de la CPUE (ton·lance ${ }^{-1}$ ) de camarón nailon por parte de la flota arrastrera con puerto base en Coquimbo. Se construyó un modelo de remoción de biomasa modificado para explicar las variaciones temporales de la CPUE basado en los conceptos de abundancia (biomasa total poblacional) y disponibilidad (biomasa que puede ser potencialmente capturada). Ambas cantidades fueron calculadas por ecuaciones, cuyas diferencias (positivas o negativas) indicaron los cambios en la disponibilidad de la biomasa a través del tiempo y el reclutamiento (magnitud y temporalidad). El modelo modificado indicó dos períodos anuales de distinta longitud en la disponibilidad de biomasa al arte de pesca. También indicó una tasa de reducción más acelerada para la biomasa que para la CPUE. Los resultados pueden influir en la determinación de la época óptima para la realización de las evaluaciones directas del recurso con fines de determinar cuotas de captura.
\end{abstract}

Palabras clave: Heterocarpus reedi, camarón nailon, biomasa, abundancia, disponibilidad, reclutamiento, capturabilidad, CPUE.

\section{A simple model for describing the biomass dynamics of the nylon shrimp Heterocarpus reedi at Coquimbo, Chile}

\begin{abstract}
A daily record of space/time dynamics was carried out on CPUE (ton $\cdot \operatorname{traw}^{-1}$ ) for the nylon shrimp trawl fishery based at Coquimbo, Chile, between September 1997 and August 2000. A modified biomass depletion model was constructed to analyze the temporal variations in CPUE based on the concepts of abundance (total population biomass) and availability (potential biomass capture). Both quantities were calculated by equations whose differences (positive and negative) indicated the changes in availability of biomass over time, as well as recruitmernt (magnitude and seasonality). The modified model suggested there were two periods annually, of different lengths, and different biomasses available with the fishing gear. The model also indicated a more rapid rate of reduction in biomass than did the CPUE. The results could influence the determination of the best season for carrying out direct resource evaluations aimed at determining catch quotas.
\end{abstract}

Key words: Heterocarpus reedi, shrimp fishery, fisheries assessment, CPUE, abundance, biomass, catch quotas, model, Chile

\section{INTRODUCCIÓN}

En Chile, existe una importante pesquería de arrastre de crustáceos entre Antofagasta y Concepción (Fig. 1). Las principales especies objetivo son el

Autor corresponsal: Eduardo P. Pérez (eperez@ucn.cl) camarón nailon (Heterocarpus reedi), langostino amarillo (Cervimunida johni) y langostino colorado (Pleuroncodes monodon), sujetas a distintos regímenes de acceso a lo largo del país (Fig. 1). En el caso particular de las capturas del camarón nailon 
se ha visto una disminución sostenida de las cuotas totales permitidas (CTP), de 10.000 ton en 1997 a 4.800 ton en 2003, una reducción equivalente al $52 \%$ (SUBPESCA, 2003). Incluso desde 2003 se decretó una veda total en la región centro-sur de Chile (VII a X Región). De acuerdo a SUBPESCA (2003), el agotamiento del stock fue producto de los altos niveles de captura observados en el pasado, los cuales habrían superado los excedentes productivos de la población. Frente a Coquimbo, la disminución en la biomasa del recurso y la consiguiente disminución en la CTP significó la desaparición de algunas empresas dedicadas a la extracción, procesamiento y exportación de camarón nailon, las cuales en los últimos años habían hecho inversiones del orden de los US\$ 2 millones, principalmente en el mejoramiento de la infraestructura, especialmente plantas de procesamiento, para adaptarlas a las normas exigidas por el mercado.

El recurso camarón nailon presenta una distribución contagiosa, caracterizada por focos de distinta abundancia distribuidos heterogéneamente dentro del rango de distribución de la especie (Acuña $e t$ al., 1996, 1998). Dada esta condición, en varias zonas del país hay focos de abundancia bien definidos. Sin embargo, la CTP anual no refleja la distribución espacial heterogénea del recurso. Así, en el 2004 se estimó una biomasa probable en la unidad de pesquería (de Antofagasta a Concepción) de 28 mil a 29 mil ton con una tasa de explotación del $18 \%$, la CTP propuesta fue de 5.130 ton, con una biomasa media de 28.500 ton (SUBPESCA, 2003). En casos donde existe una distribución espacial heterogénea del recurso la única forma de aplicar una tasa de explotación homogénea es en situaciones donde exista un conocimiento perfecto sobre la distribución espacial de la biomasa y donde la captura sea proporcional a la biomasa en cada foco de abundancia (Caddy, 1975; Seijo et al., 1997a). Otras hipótesis en torno a la asignación espacial del esfuerzo de pesca son una estrategia de búsqueda aleatoria (Magel \& Clark, 1983) o bien una explotación secuencial de los parches de abundancia (Hilborn \& Walters, 1987), sin que con estas se asegure una tasa de explotación constante espacialmente.

En el análisis espacial de la captura es relevante considerar qué focos de abundancia están siendo explotados (Sparre \& Willmann, 1993; Seijo et al., 1994). Relaciones entre distancia del puerto base, costos de transferencia (sensu Seijo et al., 1994) y rendimientos de pesca, afectan los beneficios eco- nómicos obtenidos de la captura. De esta manera, focos de alta abundancia, con altos rendimientos de ton-lance ${ }^{-1}$, pero distantes, pueden resultar económicamente menos rentables que focos de baja abundancia, pero más cercanos, de modo tal que el beneficio de pescar en ellos sea mayor (Seijo et al., 1994, 1997a, 1997b). En estos casos la concentración de la pesca y de la captura puede provocar la extinción de estos focos de abundancia, aun cuando la CTP se respete en general. Así, en la unidad de pesquería la tasa de explotación puede ser en promedio $18 \%$, pero en zonas que concentran la actividad de la flota la tasa de explotación será mayor que este porcentaje, aumentando el riesgo de extinción local del parche y menores en zonas de pesca poco atractivas económicamente.

Este análisis espacial sobre la distribución de la biomasa y del esfuerzo de pesca puede ser apoyado por la generación de modelos de evaluación y simulación diseñados a una escala local, que permitan predecir la dinámica del stock y el efecto de distintas tasas de explotación sobre éste. Este trabajo estuvo orientado a analizar la dinámica de la biomasa de la fracción del stock presente en los focos frente a Coquimbo, Chile, una de las regiones que concentra la mayor proporción de la biomasa del stock (SUBPESCA, 2003), como una plataforma inicial para el análisis espacial propuesto anteriormente.

\section{MATERIALES Y MÉTODOS}

Entre septiembre de 1997 y agosto de 2000 se realizó un seguimiento diario de la dinámica espacial y temporal de la CPUE (ton·lance ${ }^{-1}$ ) de camarón nailon por parte de la flota arrastrera con puerto base en Coquimbo, registrada a partir del análisis de las bitácoras de pesca de la flota. Esta flota opera entre Antofagasta y Concepción (Fig. 1), aunque su principal área de pesca es frente a Coquimbo (Acuña et al., 1998).

Pérez (2003) sugirió que la biomasa de camarón nailon sigue un ciclo caracterizado por cambios en la disponibilidad no atribuible al efecto de la mortalidad natural y por pesca, y que la biomasa se hace inaccesible al arte de pesca en períodos de tiempo bien definidos. De acuerdo a esto, se incorporaron dos distinciones operacionales: abundancia y disponibilidad, basadas en el concepto propuesto por Menge (1972). Se definió la abundancia como la cantidad total de biomasa presente en la población y la disponibilidad como la fracción de la abundan- 

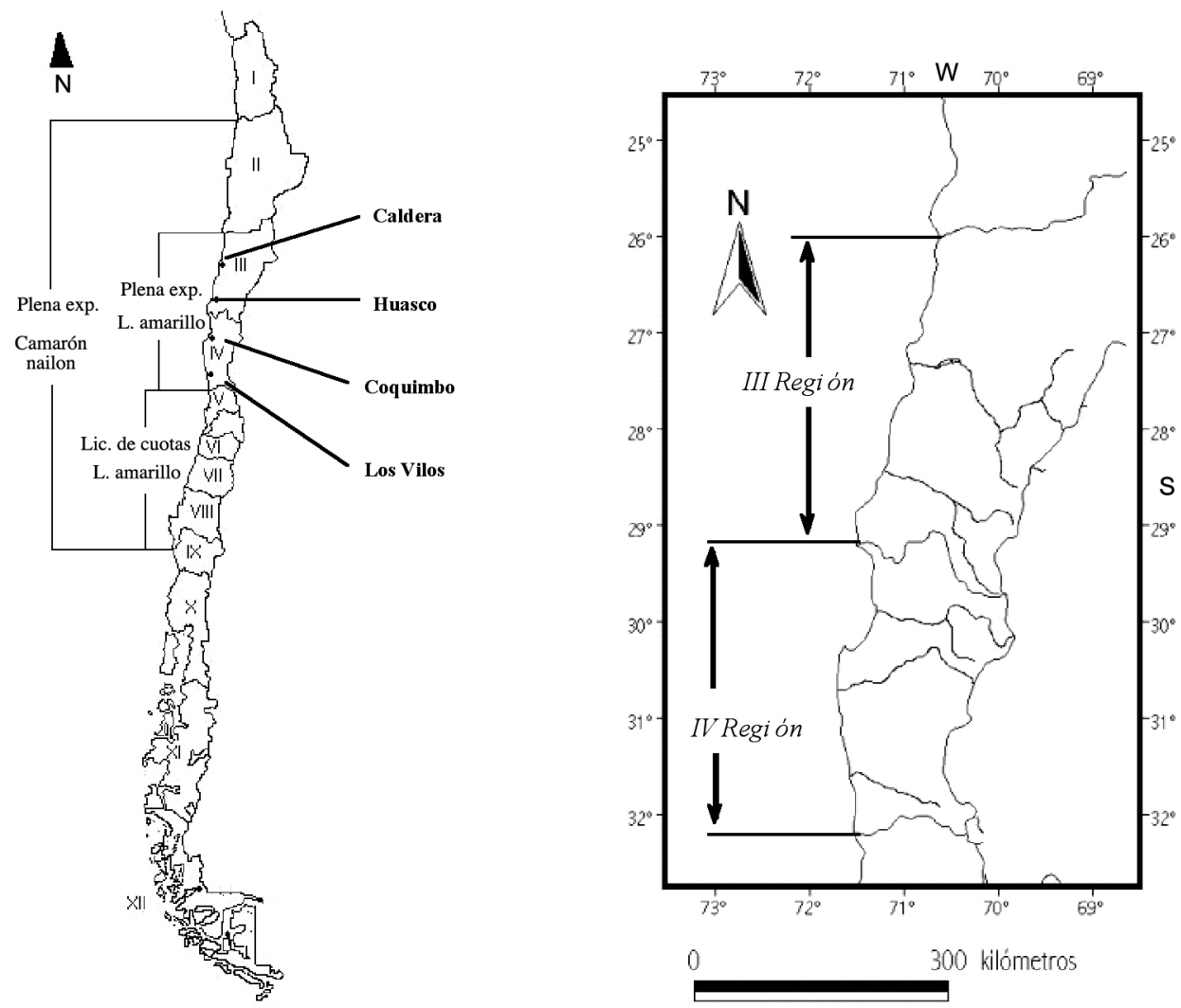

Figura 1. Localización geográfica del área de estudio, principales puertos de desembarque entre Caldera y Coquimbo y régimen de acceso para los principales recursos sometidos a explotación por la flota arrastrera con puerto base en Coquimbo (Plena exp.: Plena explotación; Lic. de cuotas: Licitación de cuotas; L. amarillo: Langostino amarillo).

Figure 1. Geographic location for the study area, main landing ports between Caldera and Coquimbo, and access regime for target species of the trawl fleet based in Coquimbo Port. (Plena exp.: Full exploited; Lic. de cuotas: Bidden quotas; L. amarillo: Squat lobster).

cia de biomasa que puede ser potencialmente capturada.

Pérez (2003) propuso que si se posee un tiempo $t$ (por ejemplo una semana) donde la disponibilidad de biomasa es máxima, entonces es posible trazar la dinámica de la biomasa tal como si no hubiese cambios en la disponibilidad del recurso (disponibili$\mathrm{dad}=$ abundancia). Esto se logra a partir de la ecuación (modificada de Kirkwood et al., 2001; Pérez \& Chávez, 2004)

$$
A B_{t}=\left(A B_{t-1}+R_{t}\right) \cdot \exp ^{-M_{s}}-C_{t}
$$

donde $\mathrm{AB}_{\mathrm{t}}$ y $\mathrm{AB}_{\mathrm{t}-1}$ es la abundancia de biomasa en la semana $t \mathrm{y} t-1$, respectivamente. $\mathrm{R}_{\mathrm{t}}$ es el recluta- miento en la semana $t, \mathrm{M}_{\mathrm{s}}$ es la mortalidad natural semanal y $\mathrm{C}_{\mathrm{t}}$ es la captura observada en la semana $t$. En el modelo la captura ocurre al final de la semana, una vez descontada la mortalidad natural y además supone la existencia de individuos de tamaño y peso promedio con el fin de darle sentido al uso de la expresión B.exp ${ }^{\text {Ms }}$ (Pérez \& Chávez, 2004) y hacerla equivalente a lo propuesto por Kirkwood $e t$ al. (2001) y Restrepo (2001) en término de número de individuos. Inicialmente, $R_{t}$ fue ajustado a cero para determinar la trayectoria de la biomasa con las pérdidas generadas exclusivamente por $\mathrm{M}_{\mathrm{s}} \mathrm{y}_{\mathrm{t}}$. Una vez descontadas estas pérdidas, el resto de la biomasa sigue estando disponible, teóricamente, al arte de pesca. A la trayectoria dinámica sin pérdida de disponibilidad se le llamó curva de abundancia (o curva control). 
Pérez (2003) mostró además que la ecuación (1) genera residuos no aleatorios en torno a la curva de ajuste con los datos observados de la pesquería, los cuales pueden ser modelados usando la ecuación

$$
\begin{gathered}
D B_{t}=\left(A B_{t-1}+R_{t}\right)^{\frac{M_{t}}{2}}-\frac{C_{t}}{2}+ \\
+\left[(a-b)\left(\operatorname{sen}\left(\left(\pi \cdot\left(\frac{t-c}{d}\right)\right)\right)\right)\right]
\end{gathered}
$$

donde $a, b, c, d$ son parámetros. La curva de biomasa calculada por la ecuación (2) fue llamada curva de biomasa disponible. Las diferencias entre las ecuaciones (1) y (2) representan los cambios en la disponibilidad del recurso (cuando la diferencia es positiva) y el reclutamiento (temporalidad e intensidad) cuando es negativa (Fig. 2). Es decir, para que exista reclutamiento es necesario que se cumpla la condición $D B_{t}>A B_{t}$.

Los parámetros de la ecuación (2) fueron calculados mediante el método de los mínimos cuadrados

$$
S S=\sum_{t=1}^{n}\left(C P U E_{o b s}-C P U E_{c a l c}\right)_{t}^{2}
$$

donde $_{\text {CPUE }}$ obs es la CPUE observada y $\mathrm{CPUE}_{\text {calc }}$ es la calculada mediante la ecuación

$$
C P U E_{t, k}=q_{k, t} \cdot D B_{t}
$$

donde $q_{k, t}$ es el coeficiente de capturabilidad en la zona de pesca $k$ en a semana $t$ y cuyos valores fueron tomados de Pérez (2003) y Pérez \& Defeo (2003), utilizando la ecuación

$$
q_{k, t}=\alpha \cdot D B_{t}^{\beta}
$$

En las ecuaciones 1 y 2 , la biomasa inicial se calculó dividiendo la CPUE observada por la capturabilidad (Pérez, 2003).

Debido a que entre Antofagasta y Coquimbo operan más embarcaciones que las que tienen puer-

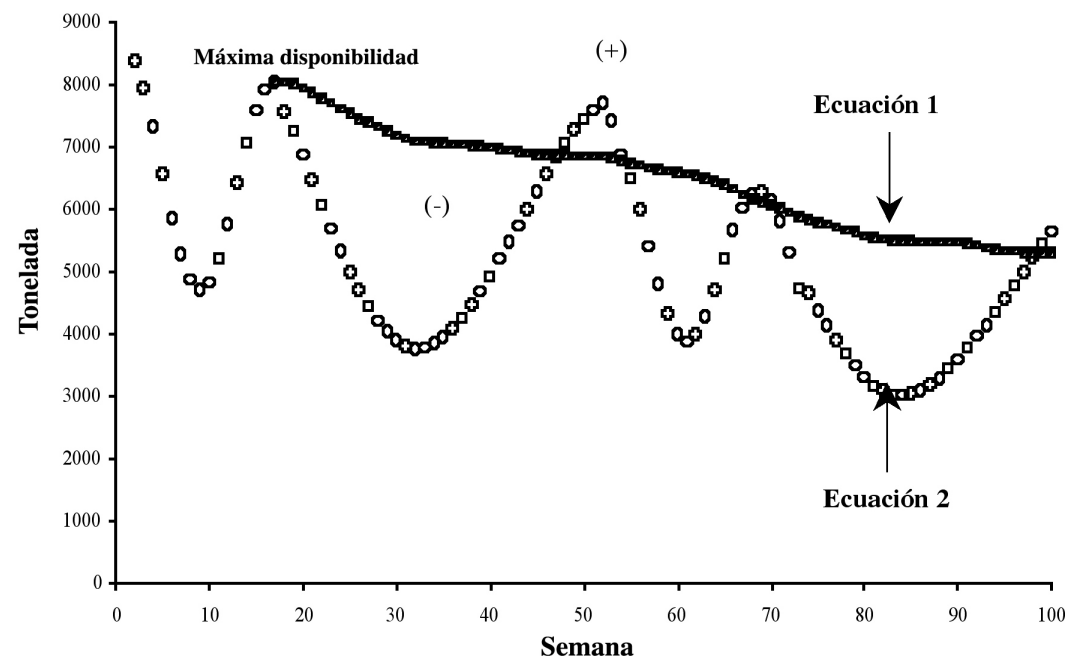

Figura 2. Representación esquemática del enfoque teórico empleado. Cuando las diferencias entre la ecuación 1 (abundancia) y 2 (disponibilidad) es positiva, tal diferencia equivale al movimiento neto de biomasa que deja de estar disponible al arte de pesca. La magnitud de la diferencia cuando ésta es negativa representa el proceso de reclutamiento.

Figure 2. Schematic representation for the theoretical approach. When difference between equation 1 (abundance) and equation 2 (availability) is positive, such difference is equivalent to net biomass movement to become unavailable to the fishing gear. When difference is negative it represents recruitment. 
to base en Coquimbo, se requirió conocer la captura total desembarcada en los puertos de esta zona: Los Vilos, Coquimbo, Caldera y Huasco (Fig. 1). Tal información está contenida en los Anuarios Estadísticos de Pesca (SERNAPESCA, 1998, 1999, 2000) en forma mensual. Para llevarla a una escala semanal se ponderó el desembarque mensual en la misma proporción que se encontró en la base de datos diarias de desembarque de la flota de Coquimbo. De esta manera se dispuso del total desembarcado en la zona de estudio.

La base de datos disponible se dividió en dos subgrupos: la primera temporada de pesca (19971998) se utilizó para estimar los parámetros del modelo y la segunda (1998-1999 y 1999-2000) con fines predictivos (validación replicativa y predictiva, respectivamente, sensu Power, 1993).

En la evaluación de la bondad del modelo se consideró el error generado, el cual pudo determinarse para cualquier tiempo $t\left(\mathrm{e}_{\mathrm{t}}\right)$ como

$$
e_{t}=P_{t}-O_{t}
$$

donde $\mathrm{P}_{\mathrm{t}}$ y $\mathrm{O}_{\mathrm{t}}$ es el valor de predicción del modelo y el valor observado al tiempo t, respectivamente. Así, el mejor ajuste es aquel que produce el menor error.

El error sistemático del modelo, cuya magnitud es una indicación de su sesgo, se calculó con la expresión,

$$
\bar{e}=\frac{\sum_{t=1}^{m} e_{n+t}}{m}
$$

donde $n$ son los valores en el subgrupo para la validación replicativa y $m$ son los valores en el subgrupo de validación predictiva. El sesgo predictivo estadísticamente significativo fue evaluado estandarizando la variable

$$
W=\frac{\sqrt{m} \cdot \bar{e}}{\sigma}
$$

para un test de dos colas la probabilidad de $\mathrm{W} \leq$ $|1,96|$ es 0,95 y valores inferiores a $\mathrm{W}>|1,96|$ son significativos (Power, 1993).

\section{RESULTADOS}

La dinámica de la biomasa y CPUE en la fase de validación replicativa pudo ser descrita por dos ciclos de diferente duración (Figs. 3a y 3b) caracterizados por los parámetros de la Tabla 1. El primer ciclo (llamado ciclo corto), en el período septiembre-diciembre (semana 1 a 17), se caracterizó por una disminución de la disponibilidad (mínima en octubre), seguido de una recuperación (máxima disponibilidad en diciembre). Esto fue seguido de un ciclo de mayor longitud (llamado ciclo largo), caracterizado por una declinación de la biomasa disponible (semana 18 a 32), llegando a un mínimo en abril (semana 32) y un posterior incremento hasta llegar a un máximo en agosto (semanas 33 a 49, Fig. 3).

Los parámetros ajustados para la fase de validación replicativa no respondieron adecuadamente en la fase de validación predictiva. El modelo ajustado presentó un nivel significativo de error y sesgo. El error fue positivo $(2,29)$, lo que representa una sobrestimación en la CPUE calculada. El sesgo fue 0,05 con un valor para el estadígrafo $\mathrm{W}$ de 156 , que es mayor al valor crítico. Esto es indicativo de un sesgo predictivo estadísticamente significativo. Ante esta situación se procedió a ajustar los parámetros de la ecuación (2) para cada una de las temporadas de pesca (Tabla 1), lo que permitió mejorar el ajuste del modelo.

La abundancia de biomasa (curva control, Fig. 4a) al inicio del período de estudio (semana 1, septiembre de 1997) fue calculada en alrededor de 5.000 ton, en tanto que la disponibilidad en el mismo tiempo fue de 4.200 ton. La abundancia de la biomasa disminuyó a 2.000 ton en agosto de 2000 (semana 143), esto es, tuvo una disminución equivalente al $60 \%$ de la biomasa al inicio de la simulación. Sin embargo, la variación porcentual en la CPUE no fue proporcional a la disminución de la abundancia en períodos de máxima disponibilidad (Fig. 4b). Mientras al inicio del período de estudio la CPUE fue de 0,59 ton $\cdot$ lance $^{-1}$ en agosto de 2000 disminuyó a 0,45 ton $\cdot$ lance ${ }^{-1}$. Es decir la disminución fue de sólo $24 \%$. En cuanto al reclutamiento, para cada estación de pesca (años 97-98 y 98-99), la diferencia entre la curva de control y la de disponibilidad permitió definir dos ventanas de tiempo para el reclutamiento, uno de menor intensidad en diciembre (Fig. 4a) y otro de mayor intensidad y prolongación entre junio y julio. 

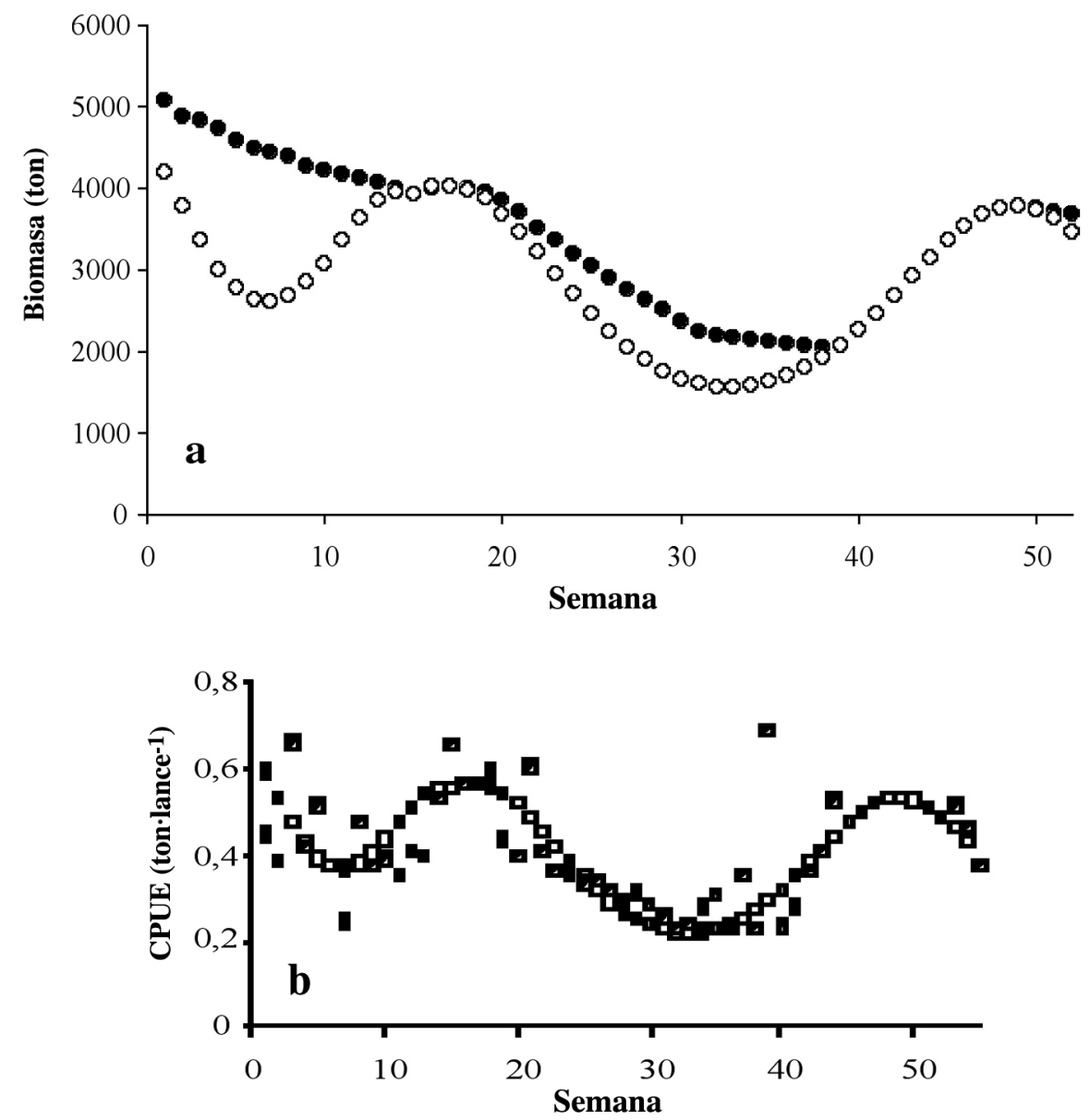

Figura 3. a) Dinámica de la biomasa de camarón nailon en Coquimbo en la temporada '97-'98: biomasa disponible (०) y biomasa control (•), b) Dinámica de la CPUE de camarón nailon en la temporada '97-'98: CPUE calculada por el modelo $(0)$ y CPUE observada $(\bullet)$.

Figure 3. a) Nylon shrimp biomass dynamic in Coquimbo during fishing season '97-'98: available biomass (O) and control biomass $(\bullet)$, b) nylon shrimp CPUE dynamic during fishing season '97-'98: CPUE estimated by the

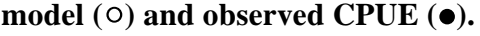



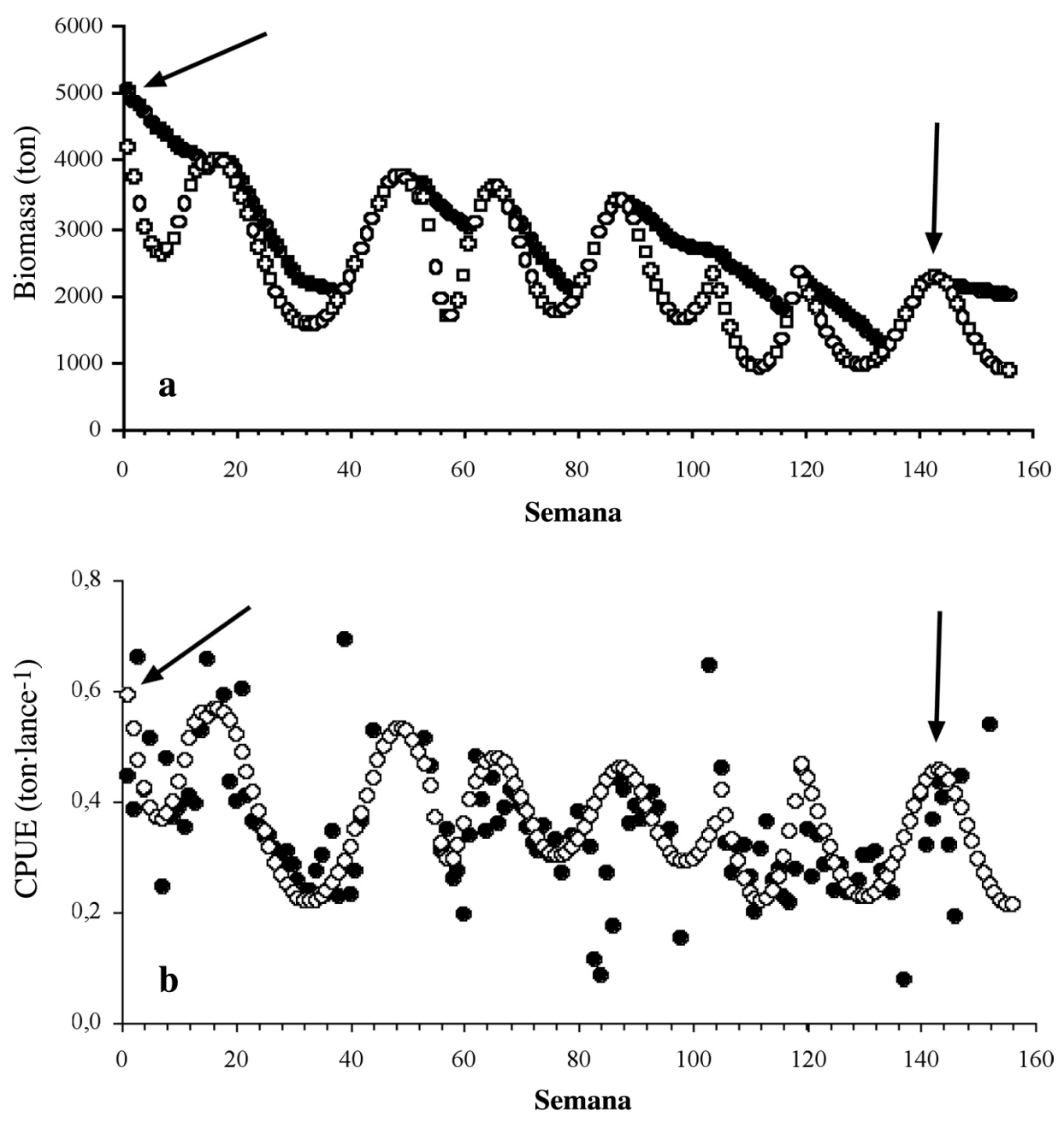

Figura 4. a) Dinámica de la biomasa de camarón nailon en Coquimbo; biomasa disponible (o) y la biomasa control $(\bullet)$ para el período de estudio, b) Dinámica de la CPUE de camarón nailon en Coquimbo: CPUE calculada por el modelo (o) y CPUE observada (•). Las flechas indican las semanas de máxima disponibilidad que son comparadas en el texto.

Figure 4. a) Nylon shrimp biomass dynamic at Coquimbo: available biomass (o) and control biomass (•) for all study period, b) nylon shrimp CPUE dynamic at Coquimbo: CPUE estimated by the model $(\bullet)$, and observed CPUE (O). Arrows show weeks with higher available biomass compared in the text. 
Tabla 1. Parámetros que definen el modelo de simulación de la pesquería de camarón nailon en Coquimbo, Chile (Pérez, 2003; Pérez \& Defeo, 2003).

Table 1. Parameters used in the simulation model of nylon fishery at Coquimbo, Chile (Pérez, 2003; Pérez \& Defeo, 2003).

\begin{tabular}{|c|c|c|c|c|c|c|c|}
\hline \multirow[t]{2}{*}{ Parámetro } & & \multicolumn{2}{|c|}{$\begin{array}{c}1^{\mathrm{a}} \text { Temporada } \\
(1997-98)\end{array}$} & \multicolumn{2}{|c|}{$\begin{array}{c}2^{\mathrm{a}} \text { Temporada } \\
(1998-99)\end{array}$} & \multicolumn{2}{|c|}{$\begin{array}{c}3^{a} \text { Temporada } \\
(1999-2000)\end{array}$} \\
\hline & & Corto & Largo & Corto & Largo & Corto & Largo \\
\hline \multirow[t]{2}{*}{$\begin{array}{l}\text { Capturabilidad } \\
\left(\text { lance }^{-1}\right)\end{array}$} & $\alpha$ & 0,000141 & 0,000141 & 0,0027 & 0,0027 & 0,00095 & 0,00095 \\
\hline & $\beta$ & 0,000 & 0,000 & $-0,368$ & $-0,368$ & $-0,201$ & $-0,201$ \\
\hline $\begin{array}{l}\text { Mortalidad } \\
\text { natural }\left(\mathrm{año}^{-1}\right)\end{array}$ & M & 0,35 & 0,35 & 0,35 & 0,35 & 0,35 & 0,35 \\
\hline Función & $\mathrm{a}$ & $-0,01$ & 0,00 & 0,00 & 0,00 & 0,00 & 0,03 \\
\hline \multirow[t]{3}{*}{ Trigonométrica } & $\mathrm{b}$ & 0,10 & 0,09 & 0,21 & 0,10 & 0,11 & 0,18 \\
\hline & c & 15 & 1,3 & 11 & 3 & 0,5 & 9 \\
\hline & $\mathrm{d}$ & 8 & -16 & 5 & -11 & -13 & -11 \\
\hline Area $\left(\mathrm{km}^{2}\right)$ & & 2069,9 & 2069,9 & 2069,9 & 2069,9 & 2069,9 & 2069,9 \\
\hline
\end{tabular}

\section{DISCUSIÓN}

El modelo elaborado, a pesar de su simplicidad y de los supuestos asociados, explicó las tendencias observadas en la pesquería durante el período de tiempo estudiado. Con relación a la biomasa de camarón nailon, Acuña \& Arancibia (1996) a través de una evaluación directa, reportaron para la zona norte de Coquimbo un total de 3.413 ton (intervalo de confianza 2.908-3.919 ton), en noviembre-diciembre de 1996. Esta estimación está en el rango calculado por el modelo para los mismos meses de la temporada 97-98, mientras que las CPUE estimadas fueron consistentes con las observaciones. Para el año 2000 la biomasa frente a Coquimbo, estimada a través de evaluación directa, fue del orden de las 3.736 ton (SUBPESCA, 2003). Aunque las diferencias respecto del modelo son de 1.736 ton, el orden de magnitud es el mismo y es consistente con la disminución de la biomasa estimada a partir de 1997 (SUBPESCA, 2001, 2003). Por otra parte, la disminución sostenida de la biomasa estimada por el modelo hacia el 2000 fue consistente con el colapso de la pesquería observado en 2001 en las empresas de Coquimbo.

Los resultados evidencian dos aspectos relevantes adicionales: a) que la CPUE en la pesquería de $H$. reedi en Coquimbo es altamente dependiente de un patrón temporal en la disponibilidad del recurso y no sólo de su abundancia a través del tiempo. Esto refuerza la necesidad que es necesario hacer la dis- tinción entre abundancia poblacional y disponibilidad de la biomasa al arte de pesca. Así, la disponibilidad disminuyó en primavera y otoño austral, mientras que aumentó hacia verano; b) que la CPUE no fue un indicador adecuado de la abundancia de biomasa de la población. Mientras la abundancia de la biomasa estimada disminuyó $61 \%$ entre el inicio y final del período analizado, en el mismo tiempo la CPUE disminuyó 24\%, es decir, la disminución de abundancia mostró una tasa más alta que la disminución de la CPUE.

En crustáceos hay evidencia de migraciones verticales y horizontales (Cruz et al., 1987; AureolesGamboa, 1995; Puga et al., 1996, entre otros), las que pueden llegar a alterar el indicador de abundancia del recurso (Taylor \& Prochaska, 1985). Aunque en el caso particular de $H$. reedi se han descrito movimientos batimétricos estacionales (Arana \& Nakanishi, 1971; SUBPESCA, 1999) representados por desplazamientos horizontales y verticales de la biomasa de camarón nailon frente a las costas de la zona de Valparaíso en Chile. Pérez (2003) al hacer un análisis por estrato de profundidad no encontró evidencia de migraciones batimétricas de $H$. reedi en Coquimbo que explicara el patrón en la disponibilidad del recurso, representada en la CPUE. Según las características biológicas de esta especie (SUBPESCA, 2003), el patrón de disponibilidad de biomasa en el tiempo coincide con la época de portación de huevos en otoño-primavera (ciclo corto) y con el período de muda (ciclo largo). De esta 
manera la disponibilidad del recurso parece asociarse a eventos reproductivos y de muda (Fig. 5), y no necesaria y exclusivamente a eventos de migraciones batimétricas, en contraste a lo señalado por Arana \& Nakanishi (1971) y por SUBPESCA (1999). Así, la muda haría a los individuos más susceptibles a la depredación. Por lo anterior, en estos períodos la necesidad de refugio disminuiría su disponibilidad al arte de pesca.

A partir de los ciclos de disponibilidad de biomasa, es crítica la época en que se llevan a cabo las evaluaciones directas con fines del cálculo de la CTP. La decisión acerca de cuándo realizar las evaluaciones directas depende en muchos casos del tiempo en que la flota o los recursos económicos estén disponibles, sin mayor consideración acerca de la disponibilidad de la biomasa en la época de la evaluación. En el camarón nailon, se sugiere que la época óptima para realizar estos cruceros de evaluación debieran coincidir con las épocas de máxima disponibilidad de biomasa, lo que ocurre alrededor de agosto y enero, época de inicio y fin del ciclo corto, respectivamente. La consecuencia de realizar las evaluaciones directas en una época de baja disponibilidad será una subestimación del tamaño del stock y una estimación errónea de la CTP para la temporada de pesca siguiente.

Respecto del error y sesgo del modelo en la fase de validación predictiva (calculados a partir de la proyección de los parámetros estimados en la validación replicativa), éste era un resultado hasta cierto punto esperable, ya que los fenómenos biológicos rara vez se repiten con exactitud en las mismas semanas año a año. Los ciclos de distinta amplitud se repitieron en cada una de las temporadas analizadas, pero en distintas semanas. Esto produjo un desfase entre lo esperado y lo observado que se tradujo en el error y sesgo estimado en el modelo. Una mención especial requiere el error inducido por el origen de la información. Es sabido (Karstegl, 2002) que el valor de la CPUE reportado en las bitácoras de pesca está subrepresentado, pero que en su trayectoria global hay correspondencia entre lo declarado y lo observado por técnicos a bordo de las embarcaciones (Karstegl, 2002). El efecto preciso de esta situación será objeto de estudios posteriores.

En el caso de $H$. reedi la CPUE no resultó ser un indicador adecuado de la abundancia relativa del recurso, mientras la abundancia de biomasa disminuyó $61 \%$, la CPUE lo hizo sólo 24\%. La razón de esto está en el valor de capturabilidad, el cual aumenta al disminuir la biomasa (Pérez \& Defeo, 2003). Esta situación ha sido reportada previamente para recursos pelágicos y demersales (MacCall, 1976; Peterman \& Sterr, 1981; Bannerot \& Austin, 1983; Crecco \& Savoy, 1985; Gordoa \& Hightower, 1991; Swain et al., 2000), peces de lagos (Langeland

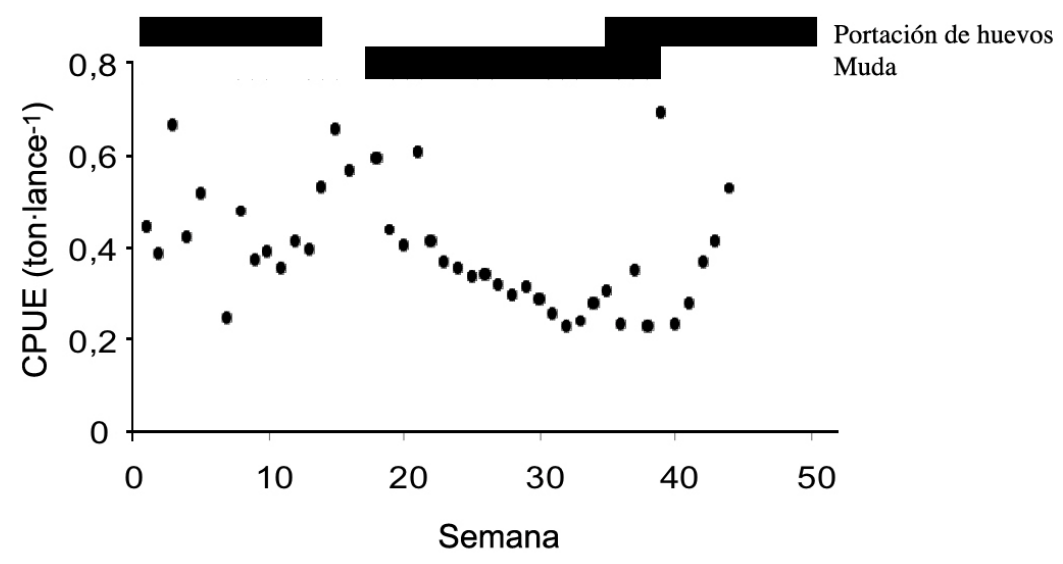

Figura 5. Relación entre la dinámica de la CPUE (temporada '97-'98) y el período reproductivo y de muda (barras de color negro), según SUBPESCA (1999).

Figure 5. Relationship between observed dynamic CPUE (fishing season '97-'98) and molting and reproductive periods (in black bars), based on SUBPESCA (1999). 
\& Pedersen, 2000), crustáceos (Ye \& Mohammed, 1999; Ziegler et al., 2002), incluso moluscos (Pérez \& Chávez, 2004). El principal efecto de esta relación de denso-dependencia es que el recurso desaparece a una tasa mayor que lo que sugiere la CPUE, aumentando el riesgo de colapso biológico para la pesquería. De lo anterior se desprende que la CPUE puede ser considerada como un estimador eficiente de la abundancia si y sólo si el parámetro $\beta$ de la ecuación 5 no es significativamente distinta de cero. Esta conclusión es similar a la expresada por Ulltang (1976) para Clupea harengus.

Finalmente, en un contexto donde la toma de decisiones y la aplicación de medidas de ordenación debe realizarse aún en ausencia de información o con una información muy limitada (Hilborn \& Peterman, 1996), el enfoque basado en un modelo de remoción modificado representó una estrategia de trabajo efectiva, llevada a cabo con información elemental, de bajo costo y que puede ser registrada por la propia industria arrastrera. Así, representa una estrategia útil y complementaria a las evaluaciones directas que involucran un mayor costo de implementación.

\section{AGRADECIMIENTOS}

El autor agradece a la Asociación de Industriales y Armadores Pesqueros, IV Región A.G., por su buena disposición en la generación de parte de la información base para el estudio. Un agradecimiento especial para tres revisores anónimos.

\section{REFERENCIAS}

Acuña, E. \& H. Arancibia. 1996. Evaluación directa del stock de camarón nailon (Heterocarpus reedi) por el método de área barrida en la zona de operación de la flota arrastrera de las regiones III y IV. Informe Final Proyecto de Investigación. Depto. Biología Marina, U. Católica del Norte, Sede Coquimbo-Depto. Oceanografía, U. de Concepción, 71 pp.

Acuña, E., E.P. Pérez \& M.T. González. 1998. Monitoreo de la pesquería de crustáceos realizada por la flota de la IV Región. Informe Final Proyecto Monitoreo de la pesquería de crustáceos. Universidad Católica del Norte-Asociación de Industriales y Armadores Pesqueros, IV Región A.G., 104 pp.
Arana, P. \& A. Nakanishi. 1971. La pesquería del camarón naylón (Heterocarpus reedi) frente a la costa de Valparaíso. Invest. Mar., Valparaíso, 2: 6192.

Aureoles-Gamboa, D. 1995. Migración batimétrica de la langostilla bentónica en la plataforma continental del Pacífico de Baja California Sur. En: D. Aurioles-Gamboa \& E. Balart (eds.). La langostilla: biología, ecología y aprovechamiento. Baja California, México, pp. 79-92.

Bannerot, S.P. \& C.B. Austin. 1983. Using frequency distribution of catch per unit of fishing effort to measure fish-stock abundance. Trans. Am. Fish. Soc., 112: 608-617.

Caddy, J.F. 1975. Spatial models for an exploited shellfish population, and its application to Georges Bank scallop fishery. J. Fish. Res. Board Can., 32: 1305-1328.

Crecco, V.A. \& T.F. Savoy. 1985. Density-dependent catchability and its potential causes and consequences on Connecticut River American shad, Alosa sapidissima. Can. J. Fish. Aquat. Sci., 42: 1649-1657.

Cruz, R., J. Baisre, E. Díaz, R. Brito, C. García, W. Blanco \& C. Carrodegas. 1987. Atlas biológicopesquero de la langosta en el archipiélago cubano. Centro de Investigaciones Pesqueras, La Habana, Cuba, 125 pp.

Gordoa, A. \& J.E. Hightower. 1991. Changes in catchability in a bottom-trawl fishery for Cape hake (Merluccius capensis). Can. J. Fish. Aquat. Sci., 48: 1887-1895.

Hilborn, R. \& C. Walters. 1987. A general model for simulation of stock and fleet dynamics in spatially heterogeneous fisheries. Can. J. Fish. Aquat. Sci., 44: 1366-1369.

Hilborn, R.M. \& R.M. Peterman. 1996. The development of scientific advice with incomplete information in the context of the precautionary approach. En: Precautionary approach to fisheries. Part 2: Scientific papers. FAO Fish. Tech. Pap., 350: 77-101.

Karstegl, A. 2002. Calibración de la CPUE y evaluación indirecta de camarón nailon (Heterocarpus reedi) y langostino amarillo (Cervimunida johni) de la pesquería de arrastre en el litoral de la III y IV Región, Chile. Tesis de Biología Marina, Universidad Católica del Norte, Coquimbo, 108 pp. 
Kirkwood, G.P., R. Auckland \& S.J. Zara. 2001. Catch effort data analysis (CEDA), Version 3.0. MRAG. London, UK.

Langeland, A. \& T. Pedersen. 2000. A 27 year study of brown trout population dynamics and exploitation in Lake Songsjøen, Central Norway. J. Fish Biol., 57: 1227-1244.

MacCall, A.D. 1976. Density dependence of catchability coefficient in the California Pacific sardine, Sardinops sagax caerulea, purse seine fishery. Calif. Coop. Oceanic Fish. Invest. Rep., 18: 136-148.

Mangel, M. \& C.W. Clark. 1983. Uncertainty, search, and information in fisheries. ICES J. Mar. Sci., 41: 93-103.

Menge, B.A. 1972. Foraging strategy of a starfish in relation to actual prey availability and environmental predictability. Ecol. Monogr., 42: 25-50.

Pérez, E.P. 2003. Análisis bio-económico de la pesquería de crustáceos en la plataforma centro-norte de Chile. Tesis de Doctorado en Ciencias. Centro de Investigación y Estudios Avanzados IPN, Unidad Mérida, México, 117 pp.

Pérez, E.P. \& O. Defeo. 2003. Time-space variation in the catchability coefficient as a function of match per unit of effort in Heterocarpus reedi (Decapoda, Pandalidae) in North-Central Chile. Interciencia, 28: 178-182.

Pérez E.P. \& J. Chávez. 2004. Modelling short-term dynamic behaviour of the surf clam (Mesodesma donacium) fishery in northern Chile using static and dynamic catchability hypotheses. Interciencia, 29: 193-198.

Peterman, R.M. \& G.J. Steer. 1981. Relation between sportfishing catchability coefficients and salmon abundance. Trans. Am. Fish. Soc., 114: 436-440.

Power, M. 1993. The predictive validation of ecological and environmental models. Ecol. Model., 68: 33-50.

Puga, R., M.E. de León \& R. Cruz. 1996. Catchability for the main fishing methods in the Cuban fishery of spiny lobster Panulirus argus (Latreille, 1804), and implications for management (Decapoda, Palinuridea). Crustaceana, 69: 703-718.

Restrepo, V. 2001. Dynamic depletion models. In: Regional workshop on the assessment of the
Caribbean spiny lobster (Panulirus argus). FAO Fish. Rep., 619: 345-356.

Seijo, J.C., J. Caddy \& J. Euan. 1994. SPATIAL: space-time dynamics in marine fisheries. A software package for sedentary species. FAO Computarized Information Series. Fisheries, 6: 116 pp.

Seijo, J.C., E.P. Pérez, M.A. Cabrera \& D. Hernández. 1997a. Riesgo e incertidumbre en el manejo de recursos vivos: un enfoque bioeconómico precautorio. Gestión de sistemas oceanográficos del Pacífico oriental. Comisión Oceanográfica Intergubernamental de la UNESCO. IOC/INF1046. pp. 203-212.

Seijo, J.C., O. Defeo \& S. Salas. 1997b. Bioeconomía pesquera: teoría, modelación y manejo. FAO Doc. Téc. Pesca, 368: 176 pp.

Servicio Nacional de Pesca (SERNAPESCA). 1998. Anuario estadístico de pesca 1997. Servicio Nacional de Pesca, Valparaíso, Chile, 306 pp.

Servicio Nacional de Pesca (SERNAPESCA). 1999. Anuario estadístico de pesca 1998. Servicio Nacional de Pesca, Valparaíso, Chile, 282 pp.

Servicio Nacional de Pesca (SERNAPESCA). 2000. Anuario estadístico de pesca 1999. Servicio Nacional de Pesca, Valparaíso, Chile, 291 pp.

Sparre, P.J. \& R. Willmann. 1993. Software for bioeconomics analysis of fisheries. BEAM 4. analytical bio-economics simulation of specestructured multi-species and multi-fleet fisheries. Volume 1: Description of model. FAO Computarized Information Series. Fisheries, 3: 186 pp.

Subsecretaría de Pesca (SUBPESCA). 1999. Cuota global anual de captura 2000 para la pesquería del camarón nailon de la II a la VIII Región. Informe Técnico (R. Pesq.), Valparaíso, $N^{\circ} 63$.

Subsecretaría de Pesca (SUBPESCA). 2001. Camarón nailon (Heterocarpus reedi). Ficha Pesquera 02, octubre de 2001.

Subsecretaría de Pesca (SUBPESCA). 2003. Cuota global anual de captura de camarón nailon entre la II y la VIII Región, año 2004. Informe Técnico de Pesca (R. Pesq.), No 102.

Swain, D.P., G.A. Poirier \& A.F. Sinclair. 2000. Effect of water temperature on catchability of Atlantic cod (Gadus morhua) to the bottom-trawl survey in the southern Gulf of St. Lawrence. ICES J. Mar. Sci., 57: 56-68. 
Taylor, T.G. \& F.J. Prochaska. 1985. Fishing power functions in aggregate bioeconomic models. Mar. Res. Econ., 2: 87-107.

Ulltang, Ø. 1976. Catch per unit of effort in the Norwegian purse seine fishery for AtlantoScandinavian herring. FAO Fish. Tech. Pap., 155: 91-101.

Recibido: 13 julio 2004; Aceptado: 21 septiembre 2005
Ye, Y. \& H. Mohammed. 1999. An analysis of variation in catchability of green tiger prawn, Penaeus semisulcatus in waters off Kuwait. US Fish. Bull., 97: 702-712.

Ziegler, P.E., S.D. Frusher \& C.R. Johnson. 2002. Space-time variation in catchability of southern rock lobster Jasus edwarsii in Tasmania explained by environmental, physiological and densitydependent processes. Fish. Res., 1472: 1-17. 